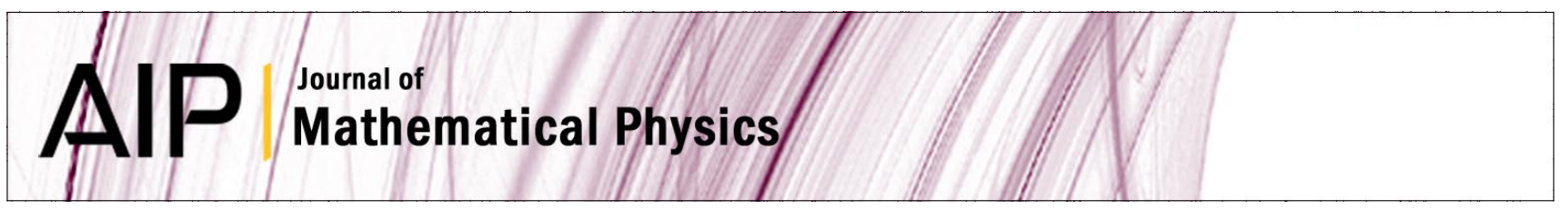

\title{
Invariant manifolds for dissipative systems
}

\author{
A. G. Ramm
}

Citation: J. Math. Phys. 50, 042701 (2009); doi: 10.1063/1.3105924

View online: http://dx.doi.org/10.1063/1.3105924

View Table of Contents: http://jmp.aip.org/resource/1/JMAPAQ/v50/i4

Published by the American Institute of Physics.

\section{Related Articles}

Attractors generated from switching unstable dissipative systems

Chaos 22, 033121 (2012)

Computation of the spectrum of spatial Lyapunov exponents for the spatially extended beam-plasma systems and electron-wave devices

Phys. Plasmas 19, 082302 (2012)

Isospectral compression and other useful isospectral transformations of dynamical networks

Chaos 22, 033118 (2012)

On the asymptotics of the Hopf characteristic function

Chaos 22, 033117 (2012)

Collision of invariant bundles of quasi-periodic attractors in the dissipative standard map

Chaos 22, 033114 (2012)

\section{Additional information on J. Math. Phys.}

Journal Homepage: http://jmp.aip.org/

Journal Information: http://jmp.aip.org/about/about_the_journal

Top downloads: http://jmp.aip.org/features/most_downloaded

Information for Authors: http://jmp.aip.org/authors

\section{ADVERTISEMENT}

The most comprehensive support for physics in any mathematical software package World-leading tools for performing calculations in theoretical physics

Your work in Maple matches how you would write the problems and solutions by hand

- State-of-the-art environment for algebraic computations in physics

The only system with the ability to handle a wide range of physics computations as well as pencil-and-paper style input and textbook-quality display of results

- Access to Maple's full mathematical power, programming language, visualization routines, and document creation tools 


\title{
Invariant manifolds for dissipative systems
}

\author{
A. G. Ramm ${ }^{\text {a) }}$ \\ Department of Mathematics, Kansas State University, Manhattan, Kansas 66506-2602, \\ USA
}

(Received 18 November 2008; accepted 2 March 2009; published online 2 April 2009)

A method is given for a study of a nonlinear evolution equation for finding "slow" invariant manifolds. The method is studied for the evolution problem $\dot{u}=-A u$ $+[(A u, u) /(u, u)] u, u(0)=u_{0}$, where $A$ is a linear, self-adjoint, possibly unbounded operator in a Hilbert space. Global existence and uniqueness of the solution to this problem are proven. Asymptotic behavior of the solution as $t \rightarrow \infty$ is studied. Analytic solution of the above nonlinear evolution problem is found. Conditions are given on the spectrum of $A$ and on the initial data $u_{0}$ for the trajectory $u(t)$ not to have a strong limit in $H$ as $t \rightarrow \infty$ and not to stay in any finite-dimensional space. In this sense the motion is chaotic. (C) 2009 American Institute of Physics.

[DOI: 10.1063/1.3105924]

\section{INTRODUCTION}

In Refs. 1 and 2 the dissipative dynamical systems, described by the evolution equation

$$
\dot{u}=-F(u), \quad u(0)=u_{0},
$$

are studied. The map $F: H \rightarrow H$ in (1) is a map in a Hilbert space $H$. This map is assumed monotone,

$$
(F(u)-F(v), u-v) \geq 0 .
$$

Physically this assumption implies dissipativity of the dynamical system. Passive nonlinear (and linear) networks give an example of a wide class of dissipative systems. ${ }^{6,7}$ A monotone operator $F$, satisfying (2), generates a semigroup $S(t)$ such that the unique solution to (1) is $u(t)=S(t) u_{0}$. A subset $H_{0}$ of $H$ is called invariant with respect to $S(t)$ if $S(t) u_{0} \in H_{0} \forall t>0$ provided that $u_{0}$ $\in H_{0}$. The problem we are interested in consists of finding invariant subsets of $S(t)$. In Ref. 1 a method for finding these subsets is proposed. These subsets are assumed to be smooth manifolds. In Ref. 16 invariant manifolds and attractors are studied for a number of nonlinear physical systems.

We describe the method for equations with linear operators. In this case the method consists of solving the problem,

$$
\dot{u}=-A u+b u, \quad b:=b(t):=b(u(t))=\frac{(A u, u)}{(u, u)}, \quad u(0)=u_{0} .
$$

Here $A \geq 0$ is a linear operator in $H$, which replaces $F$ in (1) in the case of linear equations. We assume that $A$ is closed, densely defined, self-adjoint. By $E_{s}=E((-\infty, s])$, we denote its resolution of the identity, $E_{s}=E_{s+0}$. We assume that $E_{s} u_{0} \neq 0$ for all $s \in[m, m+\delta]$, where $m$ is the infimum of the spectrum of $A$ and $\delta>0$ is a fixed number.

The idea behind Eq. (3) is simple: we subtract from $A u$ its projection onto the unit vector $u /\|u\|$. If the difference equals to zero, then we found a linear invariant subspace for $A$.

\footnotetext{
${ }^{a)}$ Electronic mail: ramm@math.ksu.edu. URL: http://www.math.ksu.edu/ramm.
} 
This idea is motivated in Ref. 1 for the case when $A$ is a matrix in a finite-dimensional space. In Ref. 1 one can find many applications of this idea to physical chemistry, polymer science, and statistical physics. In Ref. 3 and in recent paper, ${ }^{4}$ this idea is also used. However, a justification of this idea for the general case of a nonlinear monotone operator $F$ in Eq. (1) is still an open problem. In our paper this problem is solved for linear, possibly unbounded, self-adjoint operator $A \geq 0$ in an infinite-dimensional Hilbert space $H$.

It turns out that problem (3) has a unique global solution, that is, the solution $u(t)$ is defined for all $t>0$, and $u(t)$ converges strongly in $H$, as $t \rightarrow \infty$, to a limit which belongs to the eigenspace of $A$ corresponding to the minimal eigenvalue of $A$ in the segment $[m, m+\delta]$. If there are no eigenvalues of $A$ in this segment, then $u(t)$ does not converge strongly in $H$ as $t \rightarrow \infty$. If there are infinitely many eigenvalues in the segment $[m, m+\delta]$ and their infimum $m$ is an eigenvalue of $A$, then $u(t)$ converges strongly to an element of the eigenspace of $A$ corresponding to the eigenvalue $m$. Therefore, one can find an invariant with respect to $A$ linear manifold (subspace) by solving problem (3) and studying the behavior of the solution $u(t)$ as $t \rightarrow \infty$. Some of these conclusions remain valid if $A$ is not self-adjoint but its spectrum lies in an angle $|\arg \lambda|<\alpha<\pi / 2$. If $F$ is nonlinear, then one cannot, in general, expect the existence of a linear invariant manifold for $F$.

In Sec. II the existence and uniqueness of the solution $u(t)$ to problem (3) are discussed. In Sec. III the existence and nonexistence of the strong limit of the solution $u(t)$ as $t \rightarrow \infty$ are discussed as well as the time evolution of the invariant subspaces of the operator $A$. In Sec. IV a closed-form solution to problem (3) is obtained.

An interesting, unexpected, and quite novel result in our paper is an analytic solution to the Cauchy problem for the nonlinear operator, Eq. (3). This solution allows us to prove global existence and uniqueness of the solution to problem (3). Apparently, this result is not possible to obtain by the known techniques, based on the assumption that $B$ satisfies a Lipschitz condition, because the operator $B(u):=-A u+[(A u, u) /(u, u)] u$ does not satisfy this condition if $A$ is unbounded. Therefore even the local existence of the solution to problem (3) is not guaranteed $a$ priori if the operator $A$ is unbounded. Note also that even if $A \geq 0$, the operator $B$ is not necessarily monotone. Therefore the known results on the semigroups, generated by maximal monotone operators, are not applicable to problem (3) if $A$ is unbounded.

Another novel, as it seems, physical phenomenon, discussed in our paper, is the occurence of a chaotic motion of a special type in the infinite-dimensional dynamical system, described by Eq. (3). Namely, we give conditions on the spectrum of $A$ and on the initial element $u_{0}$ which are sufficient for the trajectory of the solution $u(t)$ to (3) not to have a strong limit in $H$ and not to stay in any finite-dimensional subspace of $H$. In this sense the motion is chaotic (see Remark 2 in Sec. III).

\section{EXISTENCE AND UNIQUENESS OF THE SOLUTION}

Global existence and uniqueness of the solution to problem (3) in the case when $A$ is a linear unbounded self-adjoint operator are proven in Sec. IV by a novel method, see Theorem 4.1. In Sec. II we assume that $A$ is a bounded linear self-adjoint operator and give a simple proof of the global existence and uniqueness of the solution to problem (3). This proof is based on the known techniques, in contrast to the proof in Sec. IV.

If (3) has a solution, then multiplying (3) by $u$ one gets $(\dot{u}, u)=0$. This implies $(u(t), u(t))$ $=\left(u_{0}, u_{0}\right)=$ const, $t>0$, so $\|u(t)\|=\left\|u_{0}\right\|>0, t>0$, and, therefore, the map $u_{0} \rightarrow u, u(t)=S(t) u_{0}$ is isometric.

Let $A$ be a bounded operator and $\Delta$ be a subset of the spectrum $\sigma(A)$ of the operator $A$. In the argument in Sec. III one has $\Delta=[m, m+\delta)$, where $\delta$ is a positive number. The part of $A$, which acts in the invariant subspace $E(\Delta) H$ of $A$, will be of interest for us. This part, that is, the operator $E(\Delta) A$, is bounded because $\Delta$ is a bounded interval. Therefore the assumption that $A$ is bounded is not a restrictive one for our purposes. In Sec. IV we will get rid of this assumption.

If $A$ is bounded, then problem (3) has a unique local solution because its right-hand side is Lipschitz. This solution is global because $\sup _{t>0}\|u(t)\|=\left\|u_{0}\right\|<\infty$. If $A=A^{*}$, then the solution to (3) satisfies the following relation: 


$$
u(t)=e^{-t A+\int_{0}^{t} b(s) d s} u_{0}=e^{\int_{0}^{t} b(s) d s} e^{-t A} u_{0} .
$$

\section{BASIC RESULTS}

Briefly, the basic results of this section can be described as follows.

If the infimum $m$ of the spectrum $\sigma(A)$ of the operator $A$ is an isolated eigenvalue and the projection of the initial data $u_{0}$ onto the eigenspece $H_{m}$, corresponding to this eigenvalue, is nonzero, then there exists a strong limit of the solution to problem (3) as $t \rightarrow \infty, \lim _{t \rightarrow \infty} u(t)=v$, $v \in H_{m}$, and $\|v\|=\left\|u_{0}\right\|$.

The eigenspace $H_{m}$ in this case is the slow linear invariant manifold for the dissipative dynamical system described by the problem (3). It is "slow" because the part of the solution $u(t)$ in $H_{m}$ is changing in time slower than the part corresponding to the other parts of the spectrum of A.

If the interval $\Delta=[m, m+\delta)$ consists of the points of absolutely continuous spectrum of $A$, and the projection of the initial data $u_{0}$ onto the invariant subspace $E(\Delta) H$ of $A$ is nonzero, then there does not exist strong limit in $H$ of the solution $u(t)$ to problem (3) as $t \rightarrow \infty$; the trajectory of the solution $u(t)$ does not stay in any fixed finite-dimensional subspace of $H$ or in any fixed compact subset of $H$; it does stay on an infinite-dimensional sphere $\|u(t)\|=\left\|u_{0}\right\|$ in $H$. In this sense the trajectory of the solution $u(t)$ is chaotic.

More detailed formulation of the results is given in lemmas, theorems, claims, corollaries, and remarks.

\section{A. Large-time behavior of the solution.}

Let us assume that $u_{0} \in E([m, m+\delta)) H:=E(\Delta) H$ for an arbitrarily small $\delta>0$. Then formula (4) shows that $u(t) \in E(\Delta) H$, and, by the spectral theorem for the self-adjoint operator $A$, one has

$$
b(s)=\frac{(A u(s), u(s))}{(u(s), u(s))}=\frac{\int_{m}^{m+\delta} \lambda d\left(E_{\lambda} u, u\right)}{\left(u_{0}, u_{0}\right)} \leq \frac{(m+\delta)}{\left\|u_{0}\right\|^{2}}\|E(\Delta) u\|^{2} \leq m+\delta .
$$

Lemma III.1: If $H=H_{1} \oplus H_{2}, H_{1}$ and $H_{2}$ are invariant with respect to $A$ subspaces, $H_{j}=E\left(\Delta_{j}\right) H$, $j=1,2, \Delta_{1} \cap \Delta_{2}=\emptyset$, then $u(t)=u_{1}(t) \oplus u_{2}(t), u_{j}(t) \in H_{j}$. If $\sup _{\lambda \in \Delta_{1}} \lambda:=m_{1}<m_{2}:=\inf _{\lambda_{\in} \Delta_{2}} \lambda$, then $\lim _{t \rightarrow \infty}\left\|u_{2}(t)\right\| /\left\|u_{1}(t)\right\|=0$.

Proof: Equation (3) is equivalent to the following equations:

$$
\begin{gathered}
\dot{u}_{1}=-A u_{1}+b u_{1}, \quad \dot{u}_{2}=-A u_{2}+b u_{2}, \\
u_{1}(0)=u_{01}=E\left(\Delta_{1}\right) u_{0}, \quad u_{2}(0)=E\left(\Delta_{2}\right) u_{0} .
\end{gathered}
$$

One has $\left(u_{1}, u_{2}\right)=0$ and

$$
u_{j}(t)=e^{-t A+\int_{0}^{t} b(s) d s} u_{0 j}, \quad j=1,2 .
$$

Therefore,

$$
\lim _{t \rightarrow \infty} \frac{\left\|u_{2}(t)\right\|^{2}}{\left\|u_{1}(t)\right\|^{2}}=\lim _{t \rightarrow \infty} \frac{\int_{\Delta_{2}} e^{-2 s t} d\left(E_{s} u_{0}, u_{0}\right)}{\int_{\Delta_{1}} e^{-2 s t} d\left(E_{s} u_{0}, u_{0}\right)} \leq \lim _{t \rightarrow \infty} \frac{e^{-2 m_{2} t}\left\|u_{02}\right\|^{2}}{e^{-2 m_{1} t}\left\|u_{01}\right\|^{2}}=0 .
$$

Lemma III.1 is proven. 
Lemma III.1 allows one to neglect, as $t \rightarrow \infty$, the part of the solution to problem (3), corresponding to the part of $A$ in $H_{2}=E\left(\Delta_{2}\right) H$ if inf $\Delta_{2}>\sup \Delta_{1}$. Therefore, when one studies largetime behavior of the solution to (3), one may assume without loss of generality that the operator $A$ is bounded.

Lemma 3.1 can be stated in a stronger form.

Claim 1: Suppose that $m_{1}=m_{2}:=\mu>m$ and assume that for an arbitrarily small fixed $\epsilon>0$, the spectrum of $A$ is continuous in the interval $(\mu-\epsilon, \mu+\epsilon)$. Then the conclusion of Lemma 3.1 remains valid.

Let us prove this claim. According to formula (8) it is sufficient to prove that

$$
J:=\lim _{t \rightarrow \infty} \frac{\int_{\Delta_{2}} e^{-2 s t} d\left(E_{s} u_{0}, u_{0}\right)}{\int_{\Delta_{1}} e^{-2 s t} d\left(E_{s} u_{0}, u_{0}\right)}=0 .
$$

For an arbitrarily small fixed $\epsilon>0$, Lemma 3.1 yields

$$
J_{1}:=\lim _{t \rightarrow \infty} \frac{\int_{\Delta_{2} \backslash(\mu, \mu+\epsilon)} e^{-2 s t} d\left(E_{s} u_{0}, u_{0}\right)}{\int_{\Delta_{1}} e^{-2 s t} d\left(E_{s} u_{0}, u_{0}\right)}=0 .
$$

Let $d\left(E_{s} u_{0}, u_{0}\right):=d \rho\left(s ; u_{0}\right):=d \rho(s)$. Since the spectrum of $A$ is continuous in the interval $(\mu$ $-\epsilon, \mu+\epsilon)$, one has $\lim _{\epsilon \rightarrow 0}[\rho(\mu+\epsilon)-\rho(\mu)]=0$. Thus,

$$
J_{2}:=\lim _{t \rightarrow \infty} \frac{\int_{\mu}^{\mu+\epsilon} e^{-2 s t} d\left(E_{s} u_{0}, u_{0}\right)}{\int_{\Delta_{1}} e^{-2 s t} d\left(E_{s} u_{0}, u_{0}\right)} \leq \lim _{t \rightarrow \infty} \frac{\int_{\mu}^{\mu+\epsilon} d \rho(s)}{\int_{\Delta_{1}} d \rho(s)}=[\rho(\mu+\epsilon)-\rho(\mu)] /\left\|E\left(\Delta_{1}\right) u_{0}\right\|=o(1)
$$

as $\epsilon \rightarrow 0$, where $o(1)$ is uniform with respect to $t \geq 0$.

Therefore, $J \leq o(1)$, and since $J$ does not depend on $\epsilon$ and $\epsilon$ can be taken arbitrarily small, it follows that $J=0$. Claim 1 is proven.

One may strengthen Lemma 3.1 further. Namely, the following claim holds.

Claim 2: Suppose that $\epsilon>0$ is a small fixed number. Assume that the spectrum of $A$ in the interval $[m, m+\epsilon]$ is arbitrary, containing, possibly, countably many eigenvalues $\lambda_{j}$, which may form a dense set in the interval $[m, m+\epsilon)$, so that the spectrum of $A$ in $[m, m+\epsilon)$ contains a singular component. Assume that $m$ is an eigenvalue of A, possibly of infinite multiplicity. Let $H_{m}$ be the corresponding eigenspace, $P_{m}$ be the orthogonal projector onto $H_{m}$, and assume that $P_{m} u_{0} \neq 0$. Let $H_{1}:=H_{m}$ and $H_{2}:=H_{1}^{\perp}$. Then the conclusion of Lemma 3.1 remains valid.

Proof: The idea of the proof is the same as in the proof of Lemma 3.1 and Claim 1. The role of $\Delta_{1}$ is now played by the point $m$; the denominator in (8) is $e^{-2 m t}\left\|P_{m} u_{0}\right\|^{2}>0$. In the formula analogous to (10), one takes $\Delta_{1}=\mu=m$ and replaces $\Delta_{2} \backslash[\mu, \mu+\epsilon)$ by $(m, m+\epsilon)$. The function $\left(E_{s} u_{0}, u_{0}\right):=\rho(s)$ is a monotone function. It has a unique decomposition into a sum $\rho(s)=\rho_{d}(s)$ $+\rho_{c}(s)$ of continuous part $\rho_{c}(s)$ and singular part $\rho_{d}(s)$, corresponding to the jumps of $\rho$. We want to prove that

$$
\lim _{t \rightarrow \infty} \int_{m+0}^{m+\epsilon} e^{-2 t(s-m)} d \rho(s)=0 .
$$

This follows from the Lebesgue dominant convergence theorem because the function $e^{-2 t(s-m)}$ converges to zero in measure $\rho$ as $t \rightarrow \infty$. 
Corollary 1: If $\sigma(A)$ denotes the spectrum of $A$, inf $\sigma(A)=m, m$ is an isolated eigenvalue of $A$, and $H_{1}$ is the corresponding eigenspace, $\operatorname{dim} H_{1}<\infty$, then Lemma III.1 implies that $u(t)$, the solution to (3), converges strongly, at an exponential rate, to an element of $H_{1}$. The subspace $H_{1}$ is an invariant linear manifold for problem (3).

Lemma III.2: Assume that $u_{01} \neq 0$ and $m$ is an isolated eigenvalue of $A$. Then there exists the strong limit,

$$
\lim _{t \rightarrow \infty} u(t)=u_{01} \frac{\left\|u_{0}\right\|}{\left\|u_{01}\right\|} .
$$

Proof: From Lemma III.1 it follows that

$$
\lim _{t \rightarrow \infty}\left\|u_{1}(t)\right\|=\left\|u_{0}\right\| .
$$

From (5) and (6) one gets

$$
u_{1}(t)=e^{-m t+\int_{0}^{t} b(s) d s} u_{01}, \quad\left\|u_{1}(t)\right\|^{2}=e^{-2 m t+2 \int_{0}^{t} b(s) d s}\left\|u_{01}\right\|^{2} .
$$

From (12) and (13) it follows that

$$
\lim _{t \rightarrow \infty} e^{-m t+\int_{0}^{t} b(s) d s}=\frac{\left\|u_{0}\right\|}{\left\|u_{01}\right\|} .
$$

Consequently, formula (11) is established.

Corollary 2: Under the assumption of Lemma III.1 the solution to (3) tends to an element of $H_{1}$ as $t \rightarrow \infty$, provided that $u_{01} \neq 0$.

This conclusion remains valid even if dimension of $H_{1}$ is infinite because the time evolution of every component $u_{j}(t):=\left(u(t), e_{j}\right)$, where $\left\{e_{j}\right\}$ forms an orthonormal basis of $H_{1}$, is independent of $j$.

Let us discuss the case when the point $m=\inf \sigma(A)$ is not an isolated point of spectrum.

First, let us assume that $m$ is a point of continuous spectrum of $A$ and not an eigenvalue. In this case the operator $A$ has no eigenvalues in $H_{\delta}:=E\left(\Delta_{\delta}\right) H, \Delta_{\delta}=[m, m+\delta)$. Assume that $E_{s}=E_{s+0}$ and $E\left(\Delta_{\delta}\right) u_{0} \neq 0, \forall \delta \in\left(0, \delta_{0}\right)$, where $\delta_{0}>0$ is a small fixed number.

Lemma III.3: If the spectrum of $A$ in $H_{\delta_{0}}$ is continuous and does not contain embedded eigenvalues, and if $E\left(\Delta_{\delta_{0}}\right) u_{0} \neq 0$, then there does not exist strong limit of the solution $u(t)$ to (3) as $t \rightarrow \infty$.

Proof: Suppose

$$
\lim _{t \rightarrow \infty} u(t)=f .
$$

By Lemma III.1 one may assume that $A$ is bounded, with $\|A\| \leq m+\delta_{0}$, and that $u_{0}=u_{01}$ $:=E\left(\Delta_{\delta_{0}}\right) u_{0}$. Indeed, take $u_{02}=0$, and then $u(t) \in H_{\delta_{0}}$,

$$
\|u(t)\|=\left\|u_{0}\right\|=\|f\| .
$$

If (15) holds and $A$ is bounded, then $\lim _{t \rightarrow \infty}\|A u(t)-A f\|=0$, and

$$
\lim _{t \rightarrow \infty} \frac{(A u(t), u(t))}{(u(t), u(t))}=\frac{(A f, f)}{\|f\|^{2}}:=\lambda .
$$

From Eqs. (15)-(17) and (3) the existence of the limit

$$
\lim _{t \rightarrow \infty} \dot{u}:=w=-A f+\lambda f, \quad \lambda=\frac{(A f, f)}{\|f\|^{2}},
$$

follows. 
Let us prove that $w=0$, that is, that $f$ is an eigenvector and $\lambda$ is the corresponding eigenvalue.

Since we have assumed that the set $\left[m, m+\delta_{0}\right)$ does not contain eigenvalues, this contradiction will prove that the strong limit (15) cannot exist.

To prove that $w=0$, take an arbitrary $\varphi \in H$ and let $\psi(t):=(u(t), \varphi)$. Then

$$
\lim _{t \rightarrow \infty} \psi(t)=(f, \varphi), \quad \dot{\psi}=(\dot{u}, \varphi), \quad \lim _{t \rightarrow \infty} \dot{\psi}=(w, \varphi) .
$$

Choose $\varphi=w$. Then

$$
\lim _{t \rightarrow \infty} \psi(t)=(f, w), \quad \lim _{t \rightarrow \infty} \dot{\psi}(t)=(w, w) .
$$

Let us prove that $(f, w)=0$. If this is done, then (19) implies $w=0$ and Lemma III.3 is proven.

To prove that $(f, w)=0$, rewrite Eq. (18) as

$$
w=-A f+f \frac{(A f, f)}{(f, f)}
$$

multiply (20) by $f$ and get the desired conclusion:

$$
(w, f)=0 .
$$

Relations (19) take the form:

$$
\lim _{t \rightarrow \infty} \psi(t)=0, \quad \lim _{t \rightarrow \infty} \dot{\psi}(t)=\text { const }:=c>0,
$$

if $\|w\|>0$.

Relations (22) imply const $=0$. Indeed, the second relation (22) implies

$$
\psi\left(t_{2}\right)-\psi\left(t_{1}\right)=\left(t_{2}-t_{1}\right)[c+o(1)], \text { as } t_{2}, t_{1} \rightarrow \infty .
$$

This contradicts the first relation (22) unless $c=0$. Lemma III.3 is proved.

Remark 1: In the proof of Lemma 3.4 we assumed that $A$ is a bounded self-adjoint operator. This is done without loss of generality. If $A$ is unbounded, its spectrum is a union of two nonoverlapping components, say, $[m, m+\delta)$ and $[m+\delta, \infty)$. We assume that $E([m, m+\gamma)) u_{0} \neq 0$ for some $\gamma, m<\gamma<\delta$. Then the part $u_{2}(t)$ of the solution $u(t)$, which lies in the invariant with respect to $A$ subspace $E([m+\delta, \infty)) H$, is $o\left(\left\|u_{1}(t)\right\|\right)$ as $t \rightarrow \infty$, where $u_{1}(t)$ is the part of the solution $u(t)$, which lies in the invariant with respect to $A$ subspace $E([m, m+\gamma)) H$. Therefore, one may neglect $u_{2}(t)$ and consider the part $A_{1}$ of the operator $A$ in the invariant subspace $E([m, m+\delta)) H$. In this subspace $A_{1}$ is a bounded self-adjoint operator.

Remark 2: It follows from Lemma III.3 that the trajectory $u(t), 0 \leq t<\infty$, cannot stay in a finite-dimensional subspace of $H$ or in a precompact subset of $H$. Indeed, this trajectory stays on the sphere $\|u(t)\|=\left\|u_{0}\right\|$, so it stays in a bounded set. A bounded set in a finite-dimensional subspace of $H$ is precompact. Therefore, there is a sequence $t_{n}, n=1,2, \ldots$, such that $u_{n}:=u\left(t_{n}\right) \rightarrow v$ as $n$ $\rightarrow \infty$. One may assume that $t_{n} \rightarrow \infty$ because one may consider the bounded set $u\left(t_{n}\right)$ with $t_{n}=n$, for example. The argument, used in the proof of Lemma III.3, in this case yields an existence of an eigenvalue $\lambda \in[m, m+\delta)$ of $A$, contrary to the assumption, made in Lemma III.3. Therefore the trajectory $u(t), 0 \leq t<\infty$, must travel in an infinite-dimensional subspace of $H$.

This can be interpreted as a "chaotic" behavior of the solution to the evolution problem (3) if A has continuous spectrum in the interval $[m, m+\delta)$ and does not have eigenvalues in this interval.

Let $A$ be a Schrödinger operator $-\nabla^{2}+q(x)$, and assume that $q$ is a real-valued function, $|q(x)|=O\left((1+|x|)^{-a}\right), a>2, x \in R^{3}$, and $A \geqq 0$. Then $m=0$ is the infimum of the spectrum of $A$. It is proved in Refs. 14 and 15 that under these assumptions $m$ is not an eigenvalue of $A$. Therefore, Lemma III.3 is applicable to this $A$. 
Lemma III.4: If $\lambda \in\left[m, m+\delta_{0}\right)$ is the only eigenvalue of $A$ on the interval $\left[m, m+\delta_{0}\right)$, and there exists the strong limit of $u(t)$ in $H$, that is, (15) holds, then

$$
A f=\lambda f \text {. }
$$

Proof: The conclusion of this lemma follows from Lemma III.3.

Corollary 3: Assume that there are infinitely many eigenvalues $\lambda_{1}<\lambda_{2}<\cdots$ in the interval $\left[m, m+\delta_{0}\right)$, and there are no other points of spectrum of $A$. Assume that $P_{1} u_{0} \neq 0$, where $P_{1}$ is the orthoprojector onto the eigenspace of $A$ corresponding to the eigenvalue $\lambda_{1}$. Assume also that the limit (15) exists. Then $f \in P_{1} H$.

Proof: Under the assumptions of Corollary 3 the limiting point of the eigenvalues $\lambda_{j}$ exists in $\left[m, m+\delta_{0}\right]$ and is larger than $\lambda_{1}$.

If the infimum of $\sigma(A)$ is not an eigenvalue of $A$, and $\lambda \in\left(m, m+\delta_{0}\right)$ is an eigenvalue of $A$, then the limit of $u(t)$, as $t \rightarrow \infty$, does not exist if $E([m, m+\delta)) u_{0} \neq 0$ for $m+\delta<\lambda$. This follows from Lemma 3.3. Indeed, the part of the solution $u(t)$ of problem (3), which lies in $E([m, m$ $+\delta)) H$, dominates, as $t \rightarrow \infty$, the part which lies in $E\left(\left[m+\delta, m+\delta_{0}\right)\right) H$, and by Lemma III.3 there does not exist the strong limit of $E([m, m+\delta)) u(t)$ in $H$. Thus, Corollary 3 is proven.

Will the solution $u(t)$ to (3) tend to a limit if $m=\inf \sigma(A)$ is an eigenvalue embedded in the continuous spectrum of $A$ ?

Theorem III.5: Assume that $m$ is an eigenvalue of A, and $P_{m} u_{0} \neq 0$, where $P_{m}$ is the orthoprojector onto $H_{m}$, and $H_{m}$ is the eigenspace of $A$ corresponding to the eigenvalue $m$. Then there exists the limit (15) and $f \in H_{m}$.

Proof: The conclusion of Theorem 3.5 follows from Claim 2 as follows. One writes $u(t)$ $=u_{1}(t)+u_{2}(t)$, where $u_{1}(t) \in H_{m}$ and $u_{2}(t) \perp H_{m}$ and $\lim _{t \rightarrow \infty}\left\|u_{2}(t)\right\| /\left\|u_{1}(t)\right\|=0$. Therefore there exists the limit (15) and $f=\left(\left\|u_{0}\right\| /\left\|P_{m} u_{0}\right\|\right) P_{m} u_{0}$. This result is similar to the one in Lemma 3.2.

Remark 3: Our arguments can be generalized to some classes of non-self-adjoint operators. For example, assume that $A$ is a bounded operator, with a discrete spectrum, $\operatorname{Re} \sigma(A) \geq m \geq 0$, its eigenvalues are of finite multiplicity and isolated, except, possibly, one limiting point $m \geq 0$. In this case one can write

$$
e^{-t A}=\frac{1}{2 \pi i} \int_{\mathcal{C}} e^{t s}(A+s I)^{-1} d s, \quad t>0,
$$

where the contour $\mathcal{C}$ encircles the spectrum of $A$. If the eigenvectors of $A$ form a Riesz basis of $H$, then one can solve problem (3) using expansions in eigenvectors,

$$
u(t)=\sum_{j=1}^{\infty} c_{j}(t) \varphi_{j}, \quad A \varphi_{j}=\lambda_{j} \varphi_{j} .
$$

A basis $\left\{\phi_{j}\right\}_{j=1}^{\infty}$ of $H$ is called a Riesz basis if there is a bounded linear operator $B: H \rightarrow H$, such that $B^{-1}: H \rightarrow H$ is bounded, and $B \phi_{j}=\psi_{j}$, where $\left\{\psi_{j}\right\}_{j=1}^{\infty}$ is an orthonormal basis of $H$.

Sufficient conditions for a non-self-adjoint operator $A$ to have a Riesz basis consisting of its root vectors are known (see, e.g., Refs. 5 and 9-13). Eigenfunction expansion theorems for non-self-adjoint Schrödinger operators in the whole space with complex-valued rapidly decaying potentials were obtained in Refs. 9 and 12, and for the Schrödinger operators in the exterior domains in Refs. 10, 11, and 13, one finds their applications to the eigenmode expansion and singularity expansion methods in diffraction theory.

Equation (3) implies

$$
\dot{c}_{j}=-c_{j}(t) \lambda_{j}+b(t) c_{j}(t), \quad c_{j}(0)=u_{0 j},
$$

where $u_{0 j}$ are defined by the formula 


$$
u_{0}=\sum_{j=1}^{\infty} u_{0 j} \varphi_{j},
$$

and $b(t)=b\left(t, u_{0}\right)$. Thus

$$
c_{j}(t)=e^{-\lambda_{j} t+\int_{0}^{t} b(s) d s} u_{0 j} .
$$

This formula allows one to conclude that $\lim _{t \rightarrow \infty}\left\{\left[c_{j+1}(t)\right] / c_{j}(t)\right\}=0$ if $\mathfrak{R} \lambda_{j}<\mathfrak{R} \lambda_{j+1}$.

\section{B. Evolution of the invariant subspaces.}

Let $H_{0} \subset H$ be an invariant subspace of $A$ and $u(t)$ be the solution of (3). Then

$$
u(t)=T(t) u_{0}=e^{-t A+\int_{0}^{t} b(s) d s} u_{0}, \quad b(s)=b\left(s, u_{0}\right),
$$

and $\left\|T(t) u_{0}\right\|=\|u(t)\|$. Let $\left\{v_{j}\right\}$ be an orthonormal basis of $H_{0}$. If $v_{j}(t)$ solves problem (3) with $u_{0}$ $=v_{j}$, then $\left\|v_{j}(t)\right\|=\left\|v_{j}\right\|=1, \forall t \geq 0, v_{j}(t) \in H_{0} \forall t \geq 0$ because $v_{j}(t)=e^{-t A+\int_{0}^{t} b_{j}(s) d s} v_{j}$. Since $e^{\int_{0}^{t} b_{j}(s) d s}$ is a scalar factor and $H_{0}$ is an invariant subspace of the operator $e^{-t A}$, it follows that $T(t) H_{0} \subset H_{0}$. Since $b\left(s, u_{0}\right)$ depends on $u_{0}$ nonlinearly, the mapping $T: u_{0} \rightarrow u(t)$ is nonlinear and isometric. If $P(t)$ is an orthogonal projector (projection operator) on the one-dimensional subspace, spanned by $v_{j}(t)$, that is,

$$
P_{j} f:=\left(f, v_{j}(t)\right) v_{j}(t), \quad\left\|v_{j}(t)\right\|=\left\|v_{j}(0)\right\|:=\left\|v_{j}\right\|=1,
$$

then

$$
\begin{aligned}
\dot{P}_{j} f & :=\left(f, \dot{v}_{j}(t)\right) v_{j}(t)+\left(f, v_{j}(t)\right) \dot{v}_{j}(t)=\left(f,-A v_{j}(t)+b_{j}(t) v_{j}(t)\right) v_{j}(t)+\left(f, v_{j}(t)\right)\left(-A v_{j}(t)+b_{j}(t) v_{j}(t)\right) \\
& =-P_{j} A f+2 b_{j}(t) P_{j} f-A P_{j} f .
\end{aligned}
$$

Since $f$ is arbitrary, this implies

$$
\begin{gathered}
\dot{P}_{j}(t)=-\left[P_{j}(t) A+A P_{j}(t)\right]+2 b_{j}(t) P_{j}(t), \quad P_{j}(0)=P_{j 0}, \\
P_{j 0}:=\left(f, v_{j}(0)\right) v_{j}(0) .
\end{gathered}
$$

Equation (31) is nonlinear because $b_{j}(t)$ depends nonlinearly on $P_{j}(t)$.

\section{ANALYTIC SOLUTION OF THE EVOLUTION PROBLEM}

The main result of this section is an analytic (closed form) formula for the solution to nonlinear evolution problem (3). This formula is valid for unbounded self-adjoint operators $A \geq 0$ and yields existence and uniqueness of the solution to (3).

One may assume without loss of generality that $\left\|u_{0}\right\|=\|u(t)\|=1$ because $b(t):=b(u(t))$ $=(A u, u) /(u, u)$ is invariant under the transformation $u \rightarrow \mu u$, where $\mu \neq 0$ is a constant, and (3) is also invariant under such transformation. Thus, we assume throughout this section that

$$
A=A^{*} \geq m \geq 0, \quad\left\|u_{0}\right\|=\|u(t)\|=1,
$$

and denote

$$
y(t):=(A u(t), u(t)), \quad z(t):=\int_{0}^{t} y(s) d s,
$$

where $u(t)$ is the solution to (3), so 


$$
\dot{z}=y, \quad z(0)=0 .
$$

Formula (29) can be rewritten as

$$
u(t)=e^{z} e^{-t A} u_{0} .
$$

Applying the operator $A$ to (33) and multiplying by $u$, one gets

$$
\dot{z}=e^{2 z} h(t), \quad z(0)=0 ; \quad h(t):=\left(A e^{-2 t A} u_{0}, u_{0}\right) .
$$

Integrating this equation one obtains

$$
z(t)=-\frac{1}{2} \ln \left(1-2 \int_{0}^{t} h(s) d s\right), y(t)=\dot{z}(t)=\frac{h(t)}{1-2 \int_{0}^{t} h(s) d s} .
$$

This yields an analytic formula for the solution to (3) under the assumption that $\left\|u_{0}(t)\right\|=1$,

$$
u(t)=\frac{e^{-t A} u_{0}}{\left(1-2 \int_{0}^{t} h(s) d s\right)^{1 / 2}}, \quad h(t):=\left(A e^{-2 t A} u_{0}, u_{0}\right) .
$$

Note that $2 \int_{0}^{t} h(s) d s \leq 1$, so that the above calculations are justified. Moreover, if $\left\|u_{0}\right\|=1$, then formula (36) implies that $\|u(t)\|=1$ for all $t>0$.

Let us check this statement. Using the spectral theory one gets

$$
h(t)=\int_{m}^{\infty} s e^{-2 s t} d\left(E_{s} u_{0}, u_{0}\right), \quad u_{0} \in D(A) .
$$

Therefore, using (37) and the assumption

$$
\int_{m}^{\infty} d\left(E_{s} u_{0}, u_{0}\right)=\left\|u_{0}\right\|^{2}=1
$$

one gets

$$
1-2 \int_{m}^{t} h(\tau) d \tau=1-2 \int_{m}^{\infty} s \frac{1-e^{-2 s t}}{2 s} d\left(E_{s} u_{0}, u_{0}\right)=\int_{m}^{\infty} e^{-2 s t} d\left(E_{s} u_{0}, u_{0}\right)<1, \quad \forall t>0 .
$$

Equations (36) and (38) imply that $\|u(t)\|=1$ for all $t>0$,

$$
\|u(t)\|^{2}=\frac{\left(e^{-2 t A} u_{0}, u_{0}\right)}{\int_{m}^{\infty} e^{-2 s t} d\left(E_{s} u_{0}, u_{0}\right)}=1 .
$$

We have proven that (3) has a unique global solution if $A=A^{*} \geq m \geq 0$ is an unbounded operator. 
Theorem IV.1: If $A=A^{*} \geq m \geq 0$, then problem (3) has a unique global solution for any $u_{0}$ $\in D(A)$, and this solution is given by formula (36).

From the analytic formula (36) for the solution to problem (3), which can be rewritten as

$$
u(t)=\frac{\int_{m}^{\infty} e^{-t s} d E_{s} u_{0}}{\left(\int_{m}^{\infty} e^{-2 t s} d \rho(s)\right)^{1 / 2}}, \quad \rho(s):=\left(E_{s} u_{0}, u_{0}\right),
$$

due to formula (38), one can make some conclusions about asymptotic behavior of $u(t)$ as $t$ $\rightarrow \infty$ in the case when $m$ is an eigenvalue imbedded in the continuous spectrum of $A$ on the interval $[m, m+\delta)$. Let us assume that for the given $u_{0}$ the function $\rho(s)$ is absolutely continuous on the interval $(m, m+\delta)$ and has a jump at $s=m, d \rho(s)=r(s) d s, s \in(m, m+\delta)$, where $r(s) \in L^{1}(m, m$ $+\delta)$ and $\rho(m+0)-\rho(m):=q>0$. Then the asymptotic behavior of $u(t)$ as $t \rightarrow \infty$ can be obtained by integration by parts. One has

$$
\left(\int_{m}^{\infty} e^{-2 t s} d \rho(s)\right)^{1 / 2}=\left(q e^{-2 t m}+\int_{m}^{\infty} e^{-2 t s} r(s) d s\right)^{1 / 2}=e^{-t m}\left[q^{1 / 2}+o(1)\right], \quad t \rightarrow \infty .
$$

Let us assume that the resolution of the identity $E_{s}$ is differentiable in a suitable sense on the interval $(m, m+\delta)$ with respect to an absolutely continuous measure operator projector so that

$$
\int_{m}^{\infty} e^{-t s} d E_{s} u_{0}=e^{-t m}\left[P_{m} u_{0}+o(1)\right], \quad t \rightarrow \infty,
$$

where $P_{m}$ is an orthoprojector onto the eigensubspace of $A$, corresponding to the eigenvalue $m$. Then, $\lim _{t \rightarrow \infty} u(t)=c P_{m} u_{0}$, where $c$ is a constant. This means that this eigenspace is an attractor for the infinite-dimensional dynamical system described by (3), provided that the above assumptions hold.

Concerning the differentiation of the spectral projector $E_{s}$ with respect to a scalar measure one may read, for example, Ref. 8, pp. 242-246.

Here is an example of the representation of $d E_{\lambda}$ as an integral operator. Let $A=-L$, where $L=\Delta$ is the Laplacian in $R^{3}$. Then $m=0$,

$$
d E_{\lambda} u_{0}=\int_{R^{3}} \Phi(x, y, \lambda) u_{0}(y) d y d \lambda
$$

where $u_{0} \in C_{0}^{\infty}\left(R^{3}\right)$ and $\Phi(x, y, \lambda)=\sin \left(\lambda^{1 / 2}|x-y|\right) / 4 \pi^{2}|x-y|$ (see, e.g., Ref. 8).

${ }^{1}$ Gorban, A. N. and Karlin, I. V., Invariant Manifolds for Physical and Chemical Kinetics (Springer, Berlin, 2005).

${ }^{2}$ Gorban, A. and Karlin, I., "Method of invariant manifolds and regularization of acoustic spectra," Transp. Theory Stat. Phys. 23, 559 (1994).

${ }^{3}$ Karlin, I. V. and Gorban, A. N., "Hydrodynamics from Grad's equations: What can we learn from exact solutions?" Ann. Phys. 11, 783 (2002).

${ }^{4}$ Karlin, I., Colangeli, M., and Kroeger, M., "Exact linear hydrodynamics from Boltzmann equation," Phys. Rev. Lett. 100, 214503 (2008).

${ }^{5}$ Lidskii, V., "On summability of series in principal vectors of nonselfadjoint operators," Am. Math. Soc. Transl. 40, 193 (1964).

${ }^{6}$ Ramm, A. G., in Nonlinear Electromagnetics, edited by Uslenghi, P. (Academic, New York, 1980), pp. 263-302.

${ }^{7}$ Ramm, A. G., Theory and Applications of Some New Classes of Integral Equations (Springer-Verlag, New York, 1980), Chap. 3.

${ }^{8}$ Ramm, A. G., Random Fields Estimation (World Scientific, London, 2005).

${ }^{9}$ Ramm, A. G., "Eigenfunction expansion for nonselfadjoint Schrödinger operator," Dokl. Akad. Nauk SSSR 191, 50 (1970).

${ }^{10}$ Ramm, A.G., "Eigenfunction expansions for exterior boundary problems," Diff. Eq. 7, 737 (1971).

${ }^{11}$ Ramm, A. G., "Eigenfunction expansion corresponding the discrete spectrum," Radio Eng. Electron. Phys. 18, 364 (1973). 
${ }^{12}$ Ramm, A. G., "Eigenfunction expansion for nonselfadjoint operators," Rev. Roum. Math. Pures Appl. 25, 797 (1980).

${ }^{13}$ Ramm, A. G., "Theoretical and practical aspects of singularity and eigenmode expansion methods," IEEE Trans. Antennas Propag. 28, N6, 897 (1980).

${ }^{14}$ Ramm, A. G., "Sufficient conditions for zero not to be an eigenvalue of the Schrödinger operator," J. Math. Phys. 28, 1341 (1987).

${ }^{15}$ Ramm, A. G., "Conditions for zero not to be an eigenvalue of the Schrödinger operator," J. Math. Phys. 29, 1431 (1988).

${ }^{16}$ Temam, R., Infinite-Dimensional Dynamical Systems in Mechanics and Physics (Springer-Verlag, New York, 1997). 